\title{
Incidence of metallo-beta-lactamase producing clinical isolates of Escherichia coli and Klebsiella pneumoniae in central Nepal
}

Arijit Bora*, Rajkumari Sanjana, Brajesh Kumar Jha, Surya Narayan Mahaseth and Khilasa Pokharel

\begin{abstract}
Background: The increasing and rapid spread of metallo-beta-lactamase (MBL) producing Enterobacteriaceae, particularly Escherichia coli and Klebsiella pneumoniae represents an emerging public health threat. However, limited data is available on MBL production in clinical isolates of $E$. coli and $K$. pneumoniae from Nepal. We have therefore undertaken this study to ascertain the incidence of MBL production in clinical isolates of $E$. coli and K. pneumoniae at a tertiary care teaching hospital in central Nepal.

Methods: A total of 401 consecutive, non-duplicate isolates of E. coli $(n=216)$ and K. pneumoniae $(n=185)$ were recovered from various clinical samples between July and December, 2012. These isolates were screened for the detection of carbapenemase production on the basis of their reduced susceptibility to meropenem or ertapenem by the disc diffusion method. The screened isolates were further phenotypically studied for carbapenemase production by modified Hodge test (MHT). MBL production was detected by performing combined disc test by using imipenem discs with and without ethylenediaminetetraacetic acid (EDTA), which chelates zinc required for MBL activity.

Results: Out of 216 E. coli isolates, a total of 41 isolates (18.98\%) and out of 185 K. pneumoniae isolates, a total of 39 isolates (21.08\%) were suspected to be carbapenemase- producers on the basis of their reduced susceptibility to meropenem or ertapenem. Interestingly, all the initially suspected isolates of $E$. coli and K. pneumoniae for carbapenemase production were found to be positive in both MHT and combined disc test. However, few weakly positive reactions were observed in MHT. All the MBL producing isolates were multidrug-resistant (MDR). In addition, $75.60 \%$ E. coli and 71.79\% of K. pneumoniae isolates producing MBL were found to be "pandrug- resistant".

Conclusions: Our findings showed MBL production in a considerable number of E. coli and K. pneumoniae isolates with MDR and pandrug-resistant phenotypes. Combined disc method can provide a sensible choice for phenotypic detection of MBL production in clinical microbiology laboratories as detection of MBL in bacterial isolates is indispensable for establishing the effective antibiotic policies and infection control strategies in the hospital setting.
\end{abstract}

Keywords: Escherichia coli, Klebsiella pneumoniae, Metallo-beta-lactamase, Carbapenemase, Multidrug-resistant, Pandrug-resistant

\section{Background}

Over the past few years, metallo-beta-lactamase (MBL) producing gram negative bacteria are being reported with increasing frequency from several parts of the world and have emerged as a most widespread and clinically significant carbapenem resistance mechanisms [1]. MBL producing bacteria can hydrolyze a wide range of beta-

\footnotetext{
* Correspondence: arijitbora07@gmail.com

Department of Microbiology, College of Medical Sciences, P.O. Box 23, Bharatpur, Nepal
}

lactam antibiotics including penicillins, cephalosporins, carbapenems, cephamycins, but lack the ability to hydrolyze aztreonam. Moreover, their catalytic activities are generally not neutralized by commercially available $\beta$ lactamase inhibitors such as clavulanate, tazobactam, and sulbactam [2]. These enzymes belong to Ambler class $B$ beta-lactamases based on their amino acid sequence homology and to group 3 according to the Bush classification based on their substrate and inhibitor profiles $[3,4]$. MBLs require zinc-ions to catalyze the hydrolysis 
of beta-lactam antibiotics and due to the dependence on zinc-ions, MBL catalysis is inhibited in presence of metal-chelating agents like ethylenediaminetetraacetic acid (EDTA) [5].

MBLs are encoded either by genes that are part of the chromosome in some bacterial species (resident MBLs), or by heterologous genes acquired by horizontal gene transfer (acquired MBLs) [6]. The more geographically widespread MBLs include imipenemase (IMP), Verona integron-encoded metallo-beta-lactamase (VIM), and New Delhi metallo-beta-lactamase (NDM) [5]. MBLs were common in Pseudomonas aeruginosa and Acinetobacter spp., but more recently have emerged at an increasing rate among the members of Enterobacteriaceae [7].

Although, production of MBLs in clinical isolates represent a serious therapeutic challenge, till date clinical data are surprisingly inadequate with regard to their incidence in the members of Enterobacteriaceae, particularly from Nepal. This prompted us to conduct the present study to determine the incidence of MBL production phenotypically among the isolates of two important members of Enterobacteriaceae, Escherichia coli and Klebsiella pneumoniae as well as their antibiotic susceptibility pattern to formulate an antimicrobial policy on the basis of the local epidemiological data.

\section{Methods}

\section{Bacterial isolates}

A total of 401 consecutive, non-duplicate isolates of E. coli $(\mathrm{n}=216)$ and $K$. pneumoniae $(\mathrm{n}=185)$ were recovered from various clinical samples in clinical microbiology laboratory of College of Medical Sciences, a tertiary care 1050-bed teaching hospital in central Nepal. Samples were obtained from the hospitalized patients of different hospital units between July and December, 2012. Distribution of the sources of these isolates was: urine $(n=275)$, sputum $(n=62)$, pus $(n=39)$ and blood $(n=25)$. The samples were processed for isolation and identification based on standard laboratory techniques [8]. This study was carried out with consent from the Institutional Review Committee (IRC) of College of Medical Sciences, Bharatpur, Nepal.

\section{Antimicrobial susceptibility testing}

Antimicrobial sensitivity testing was performed on Mueller-Hinton agar (MHA) plates by Kirby-Bauer disc diffusion method, according to Clinical Laboratory Standards Institute (CLSI) guidelines [9]. The antibiotics used were: ampicillin $(10 \mu \mathrm{g})$, cephalexin $(30 \mu \mathrm{g})$, cefotaxime $(30 \mu \mathrm{g})$, ceftazidime $(30 \mu \mathrm{g})$, cefpodoxime $(10 \mu \mathrm{g})$, ceftriaxone $(30 \mu \mathrm{g})$, cefepime $(30 \mu \mathrm{g})$, aztreonam $(30 \mu \mathrm{g})$, cefoxitin $(30 \mu \mathrm{g})$, piperacillin/tazobactam $(100 / 10 \mu \mathrm{g})$, imipenem $(10 \mu \mathrm{g})$, meropenem $(10 \mu \mathrm{g})$, ertapenem $(10 \mu \mathrm{g})$, co-trimoxazole $(25 \mu \mathrm{g})$, ciprofloxacin $(5 \mu \mathrm{g})$, levofloxacin $(5 \mu \mathrm{g})$, gentamicin $(10 \mu \mathrm{g})$, amikacin $(30 \mu \mathrm{g})$, tigecycline $(15 \mu \mathrm{g})$ and colistin $(10 \mu \mathrm{g})$. All the antibiotic discs and the media were procured from Hi-media, Mumbai, India. E. coli ATCC 25922 was used as quality controls in antibiotic susceptibility testing. The results were interpreted as per CLSI guidelines [9] except, tigecycline and colistin. The results for colistin were interpreted by following the criteria proposed by Galani et al. [10] and for tigecycline by following the breakpoints for Enterobacteriaceae as suggested by Food and Drug Administration (FDA). The minimum inhibitory concentration (MIC) values for imipenem, meropenem, ertapenem, tigecycline and colistin were determined by using Etest strips (bioMerieux, France) as per the manufacturer's protocol.

\section{Screening for carbapenemase production}

By disc diffusion, each of the isolate with a reduced susceptibility to meropenem or ertapenem (inhibition zone diameter of $\leq 21 \mathrm{~mm}$ ) was screened for the production of carbapenemase according to the CLSI guidelines [9].

\section{Detection of carbapenemase production}

The phenotypic detection of the carbapenemase production was performed by the modified Hodge test (MHT) as described by CLSI [9]. Briefly, a 0.5 McFarland standard suspension of E. coli ATCC 25922 was prepared in $5 \mathrm{ml}$ peptone water and diluted $1: 10$ by adding $0.5 \mathrm{ml}$ of the 0.5 McFarland to $4.5 \mathrm{ml}$ of peptone water. A lawn of the 1:10 dilution of E. coli ATCC 25922 was prepared on a MHA plate as for the routine disc diffusion procedure. The plate was allowed to dry 3 to 10 minutes. A $10 \mu \mathrm{g}$ ertapenem disc was placed in the centre of the test plate and the test organism was streaked in a straight line from the edge of the disc to the edge of the plate. Three organisms were tested on the same plate with one drug. The plate was incubated at $37^{\circ} \mathrm{C}$ in ambient air for $16-24$ hours. After incubation, a positive MHT test was indicated by a clover leaf-like indentation of the E. coli ATCC 25922 growing along the test organism growth streak within the disc diffusion zone and a negative MHT test was indicated by no growth of the E. coli ATCC 25922 along the test organism growth streak within the disc diffusion zone (Figure 1).

\section{Detection of metallo-beta-lactamase production}

MBL production was detected by performing combined disc test described by Franklin et al. [11] in all carbapenemase screening positive isolates. In this test, two imipenem discs $(10 \mu \mathrm{g})$, one containing $10 \mu \mathrm{l}$ of $0.1 \mathrm{M}(292 \mu \mathrm{g})$ anhydrous EDTA (Sigma Chemicals, St. Louis, MO) were used. They were placed on a MHA plate inoculated with 0.5 McFarland suspension of the test isolate. Plates were incubated for $16-18$ hours at $35^{\circ} \mathrm{C}$. After incubation, the diameter of inhibition zones was measured. An increase in zone diameter of $>4 \mathrm{~mm}$ around the imipenem-EDTA 


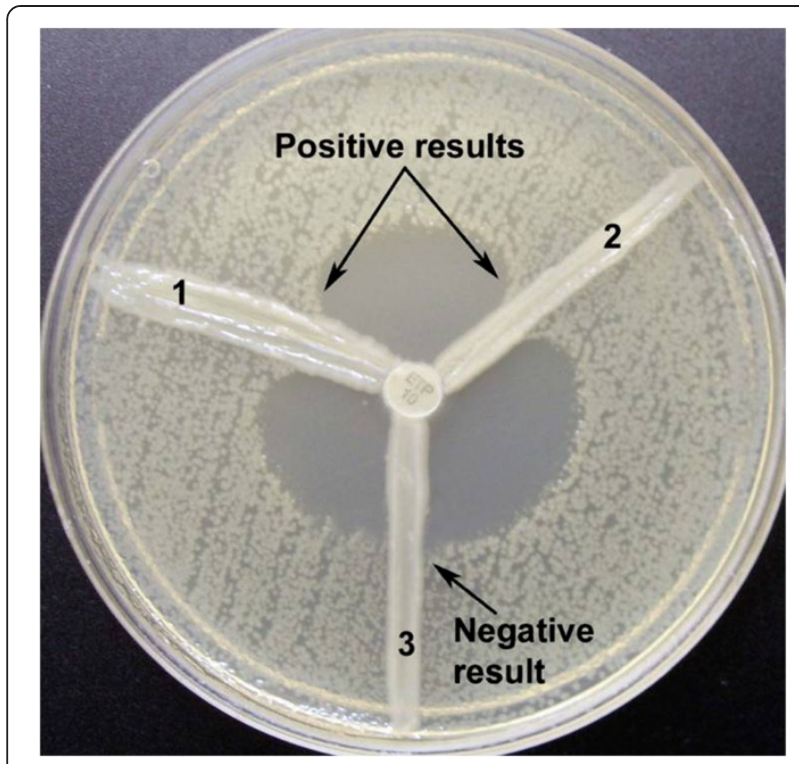

Figure 1 Modified Hodge test using a $10 \mu \mathrm{g}$ ertapenem disc. Isolates 1 and 2 produce carbapenemase and are positive by this test. Isolate 2 does not produce carbapenemase and is negative by this test.

disc compared to that of the imipenem disc alone was considered positive for MBL production (Figure 2).

E. coli strain NDM-1 EC27 (positive for NDM-1; kindly provided by Prof. Giasuddin Ahmed) was used as positive control in MHT and combined disc test. E. coli ATCC 25922 was used as negative control for both the tests.

\section{Results}

Out of 216 E. coli isolates, a total of 41 isolates (18.98\%) and out of $185 \mathrm{~K}$. pneumoniae isolates, a total of 39 isolates $(21.08 \%)$ were initially screened for carbapenemase

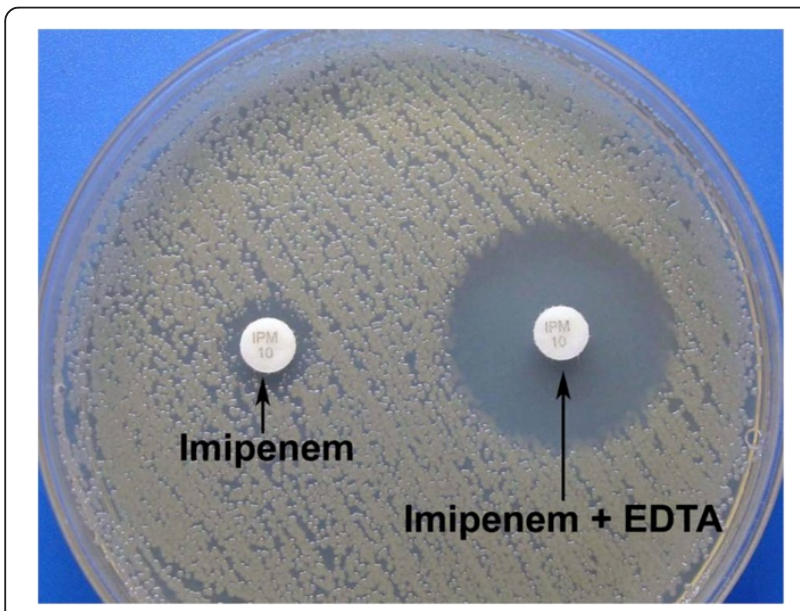

Figure 2 Positive combined disc test for detection of MBL producer by use of EDTA. The test isolate shows a zone diameter of $>4 \mathrm{~mm}$ around the imipenem-EDTA disc compared to that of the imipenem disc alone. production on the basis of their reduced susceptibility to meropenem or ertapenem by disc diffusion test. All the screening positive isolates of $E$. coli and $K$. pneumoniae were found to be positive for carbapenemase production by MHT. However, 3 (7.31\%) isolates of E. coli and 4 (10.26\%) isolates of $K$. pneumoniae gave weakly positive reactions in $\mathrm{MHT}$. Interestingly, all the screening positive isolates of $E$. coli and $K$. pneumoniae showed fairly positive results in combined disc test for MBL production. The age of the patients with MBL positive isolates ranged from 10 days to 72 years and the male to female ratio was 1.15:1.

Among the MBL positive isolates of E. coli, $53.56 \%$ $(22 / 41)$ isolates were recovered from the patients admitted to intensive care units (ICU) and 46.34\% (19/41) isolates were recovered from the patients admitted to different hospital wards (Figure 3). Likewise, among the MBL positive isolates of $K$. pneumoniae, 58.97\% (23/39) isolates were recovered from the patients admitted to ICU and $46.15 \%$ (18/39) isolates were recovered from the patients admitted to different hospital wards (Figure 3). Samplewise distribution of MBL producing isolates of $E$. coli and K. pneumoniae are shown in Figure 4.

By disc diffusion susceptibility testing, each of the MBL producing isolate of E. coli and K. pneumoniae was found to be resistant to all beta-lactam antibiotics including imipenem, meropenem, ertapenem, aztreonam as well as beta-lactam/beta-lactamase combination used in this study. Susceptibility pattern of these isolates differed with different classes of non- beta-lactam antibiotics (Table 1). All the MBL producing isolates are found to be multidrug-resistant (MDR) i.e., resistant to three or more classes of antimicrobials. In addition, $75.60 \%$ of $\mathrm{MBL}$ producing $E$. coli isolates and $71.79 \%$ of $K$. pneumoniae MBL producing E. coli isolates were "pandrug- resistant" i.e., resistant to 7 antimicrobial agents (cefepime, ceftazidime, imipenem, meropenem, piperacillin/tazobactam, ciprofloxacin, and levofloxacin). However, each of the

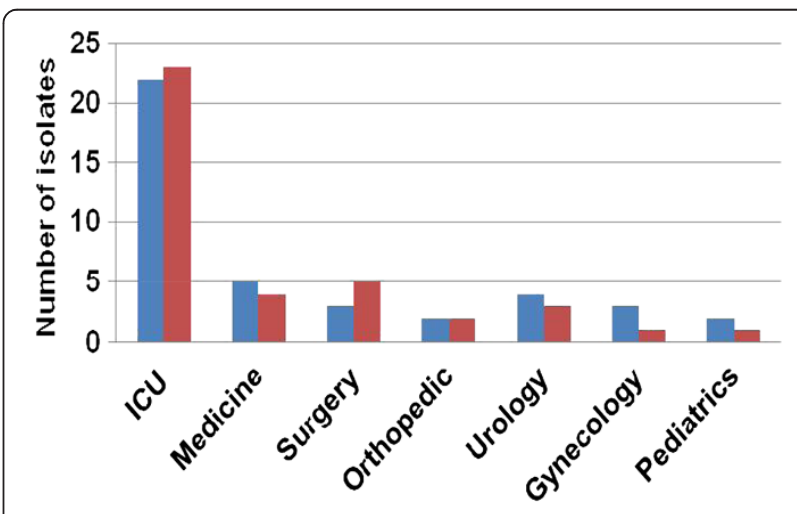

Figure 3 Distribution of MBL producing isolates of $E$. coli and K. pneumoniae in different hospital units. 


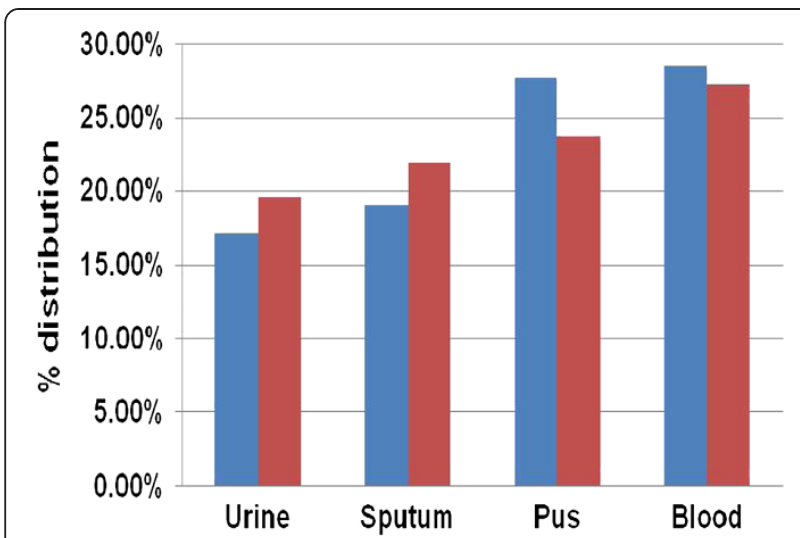

Figure 4 Sample-wise distribution of MBL producing isolates of E. coli and K. pneumoniae.

MBL producing isolate of E. coli and K. pneumoniae were found to be sensitive to tigecycline and colistin by disc diffusion test. The MIC values of MBL producing $E$. coli and $K$. pneumoniae isolates were found to vary widely for the different carbapenems used in the study (Table 2). All
MBL producing isolates showed MIC values in effective range for tigecycline and colistin.

\section{Discussion}

The increasing and rapid spread of MBL producing Enterobacteriaceae, particularly E. coli and K. pneumoniae constitutes a serious threat to public health worldwide. The present study indicated a high incidence of $\mathrm{MBL}$ producing E. coli (18.98\%) and K. pneumoniae (21.08\%) in different clinical samples. A previous study from an another tertiary care hospital in Nepal reported comparatively lower incidence of MBL producing gram negative bacteria (1.3\%) in lower respiratory tract specimens [12]. However, a recent study from Nepal addressed the issue of increasing incidence of MBL producing $K$. pneumoniae (18.2\%) in tracheal aspirate samples [13]. Several recent studies from other parts of Asia also demonstrated increasing incidence of MBL production in Enterobacteriaceae isolates [14-16]. In general, production of MBL in Enterobacteriaceae isolates currently follows an increasing prevalence pattern and the prevalence rate may vary greatly in different geographical areas and from institute to institute. In our hospital setting, extended spectrum

Table 1 In vitro susceptibility pattern of MBL producing and non-MBL-producing isolates of $E$. coli and $K$. pneumoniae by disc diffusion test

\begin{tabular}{|c|c|c|c|c|}
\hline \multirow{3}{*}{ Antibiotics tested } & \multicolumn{4}{|c|}{$\%$ sensitive } \\
\hline & \multicolumn{2}{|c|}{ E. coli $(n=216)$} & \multicolumn{2}{|c|}{ K. pneumoniae $(n=185)$} \\
\hline & Non-MBL producer $(n=175)$ & MBL producer $(n=41)$ & Non-MBL producer $(n=146)$ & MBL producers $(n=39)$ \\
\hline Ampicillin $(10 \mu \mathrm{g})$ & 2.85 & 0.00 & 0.00 & 0.00 \\
\hline Cephalothin $(30 \mu \mathrm{g})$ & 16.57 & 0.00 & 13.69 & 0.00 \\
\hline Cefpodoxime $(10 \mu \mathrm{g})$ & 35.42 & 0.00 & 33.56 & 0.00 \\
\hline Ceftazidime $(30 \mu \mathrm{g})$ & 40.57 & 0.00 & 37.67 & 0.00 \\
\hline Cefotaxime $(30 \mu \mathrm{g})$ & 38.85 & 0.00 & 39.72 & 0.00 \\
\hline Ceftriaxone $(30 \mu \mathrm{g})$ & 43.42 & 0.00 & 42.46 & 0.00 \\
\hline Cefepime $(30 \mu \mathrm{g})$ & 46.85 & 0.00 & 44.52 & 0.00 \\
\hline Aztreonam $(30 \mu \mathrm{g})$ & 40.0 & 0.00 & 41.09 & 0.00 \\
\hline Cefoxitin $(30 \mu \mathrm{g})$ & 64.57 & 0.00 & 69.18 & 0.00 \\
\hline $\begin{array}{l}\text { Piperacillin/tazobactam } \\
(100 / 10 \mu \mathrm{g})\end{array}$ & 82.85 & 0.00 & 77.39 & 0.00 \\
\hline Imipenem $(10 \mu \mathrm{g})$ & 100.00 & 0.00 & 100.00 & 0.00 \\
\hline Meropenem $(10 \mu \mathrm{g})$ & 100.00 & 0.00 & 100.00 & 0.00 \\
\hline Ertapenem $(10 \mu \mathrm{g})$ & 100.00 & 0.00 & 100.00 & 0.00 \\
\hline Ciprofloxacin $(5 \mu \mathrm{g})$ & 64.00 & 7.31 & 67.12 & 10.25 \\
\hline Levofloxacin $(5 \mu \mathrm{g})$ & 85.14 & 24.39 & 87.67 & 28.20 \\
\hline Co-Trimoxazole & 61.14 & 0.00 & 65.75 & 7.69 \\
\hline Gentamicin $(10 \mu \mathrm{g})$ & 72.57 & 17.07 & 70.54 & 15.38 \\
\hline Amikacin $(30 \mu \mathrm{g})$ & 80.57 & 21.95 & 78.08 & 20.51 \\
\hline Tigecycline $(15 \mu \mathrm{g})$ & 100.00 & 100.00 & 100.00 & 100.00 \\
\hline Colistin $(10 \mu \mathrm{g})$ & 100.00 & 100.00 & 100.00 & 100.00 \\
\hline
\end{tabular}


Table 2 MIC ranges found for MBL producing $E$. coli and $K$. pneumoniae isolates by using Etest strips

\begin{tabular}{lccccc}
\hline MBL producing & \multicolumn{3}{c}{ MIC range $(\boldsymbol{\mu g} / \mathbf{m L})$} \\
\cline { 2 - 5 } isolates & Imipenem & Meropenem & Ertapenem & Tigecycline & \multicolumn{1}{c}{ Colistin } \\
\hline E. coli & $2.0-8.0$ & $3.0-16$ & $8.0->32$ & $0.125-0.75$ & $0.125-0.5$ \\
K. pneumoniae & $2.0->32$ & $2.0->32$ & $6.0->32$ & $0.38-2.0$ & $0.125-0.5$ \\
\hline
\end{tabular}

beta-lactamases are prevalent in E. coli and K. pneumoniae isolates and there is a gradual rise in the use of carbapenems, which could be a major cause of MBLmediated resistant.

The majority of MBL producing isolates of $E$. coli (53.56\%) and K. pneumoniae (58.97\%) were from patients admitted to ICU. The ICU has been described as a factory for creating, disseminating, and amplifying antimicrobial resistance [17]. Among the four different sources of samples (urine, sputum, pus and blood), we observed highest incidence of $\mathrm{MBL}$ producing isolates in blood samples and lowest incidence in urine samples for the both pathogens. This observation also indicated the greater use of carbapenems in bloodstream infections in our setting.

Detection methods for carbapenemase production include MHT, double disc test, blood agar combined disc assay, PCR amplification, and DNA sequencing [18]. CLSI recommends the MHT as a phenotypic confirmatory test for detection of carbapenemase production in Enterobacteriaceae isolates with elevated MICs for carbapenems or reduced inhibition zones in disc diffusion susceptibility testing, due to its acceptable sensitivity and specificity for carbapenemase detection. However, the sensitivity and the specificity of this test for detecting low-level MBL production are not known [9]. In this study, out of $80 \mathrm{E}$. coli and K. pneumoniae isolates initially screened for carbapenemase production, 73 (91.25\%) isolates exhibited fairly positive results in MHT, whereas 7 (8.75\%) isolates exhibited weakly positive results in MHT. Different studies also reported the occurrence of weakly positive results for the MHT in the detection of MBL producing Enterobacteriaceae [19,20].

On the other hand, the combined disc test performed to detect MBL production was found to be highly sensitive (100\%) as this test showed positive results with all the isolates of E. coli and K. pneumoniae, which were initially screened for carbapenemase production by disc diffusion test. The difference in the zone diameter was fairly more than $4 \mathrm{~mm}$ for each of isolate tested by combined disc test. Franklin et al. [11] also reported $100 \%$ sensitivity of combined disc test in the detection of MBL in gram negative bacilli. These results suggest that combined disc test can be used as a convenient method for detection of MBL producing E. coli and K. pneumoniae isolates in clinical laboratory on a daily basis. Conversely, the combined disc test would not detect class A carbapenemases such as GES, KPC and SME, while the MHT would do so.
Besides, molecular detection of carbapenemase genes is an interesting alternative but remains costly and requires substantial expertise.

MBL producing bacterial isolates can confer resistance to carbapenems and all beta-lactam agents except aztreonam although coexistence of other resistance mechanisms such as AmpC type beta-lactamases or ESBLs render them resistant to aztreonam [20]. Likewise, all the isolates of E. coli and K. pneumoniae with MBL production in the present study were found to be resistant to all three carbapenems (imipenem, meropenem and ertapenem). These isolates also exhibited a high level of resistance to the penicillins, the third and fourth generation cephalosporins, cephamycin, and aztreonam, as well as to the beta-lactam/beta-lactamase inhibitor combination tested in the study. These findings are similar with other reports $[21,22]$, whereas in few reports MBL producing Enterobacteriaceae isolates were found to be susceptible to various carbapenems as well as to piperacillin/tazobactam by disc diffusion testing [23].

In this study, we observed that MBL production in $E$. coli and $K$. pneumoniae isolates was not always associated with elevated MIC values for the different carbapenems tested and these MIC values varied greatly among the MBL producing isolates. This variation in the MIC values for the different carbapenems may be influenced by several factors, such as the type and the expression of the carbapenemase enzyme, the bacterial species and the presence of other resistance mechanisms (e.g., Extended spectrum and AmpC beta-lactamases, reduced permeability and/or efflux pumps) [24]. Among the MBL producing isolates, the lowest MIC value for imipenem, meropenem and ertapenem were $2 \mu \mathrm{g} / \mathrm{mL}, 3 \mu \mathrm{g} /$ $\mathrm{mL}$ and $8 \mu \mathrm{g} / \mathrm{mL}$ respectively for $E$. coli and $2 \mu \mathrm{g} / \mathrm{mL}$, $2 \mu \mathrm{g} / \mathrm{mL}$ and $6 \mu \mathrm{g} / \mathrm{mL}$ respectively for $K$. pneumoniae isolates. The highest $\mathrm{MIC}$ value of $>32 \mu \mathrm{g} / \mathrm{mL}$ was observed for all the three carbapenems in $12.82 \% \mathrm{MBL}$ producing isolates of $K$. pneumoniae. Conversely, $7.3 \%$ of $\mathrm{MBL}$ producing isolates of $E$. coli showed the highest MIC value of $>32 \mu \mathrm{g} / \mathrm{mL}$ only for ertapenem. Carbapenem MICs for carbapenemase producers may vary within a broad range of values, and even lay within the susceptibility range, as defined by either the current CLSI breakpoints. Indeed, such low levels of resistance to carbapenems have often been observed in Enterobacteriaceae producing carbapenemases of different types [25]. Therefore, any Enterobacteriaceae isolate with reduced susceptibility to carbapenems 
either by disc diffusion or MIC testing should be tested for carbapenemase production.

It has been observed that carbapenemase producers are usually associated with many other non beta-lactam resistance determinants, which give rise to "MDR and pandrug-resistant" isolates [26]. We also observed 100\% MBL producers as "MDR" as well as $75.60 \%$ of $E$. coli and $71.79 \%$ of $K$. pneumoniae isolates producing MBL were "pandrug-resistant". These results highlighted a potential threat to hospitalized patients by limiting the therapeutic options. Among the MBL producing isolates, comparatively lower resistant rate was for levofloxacin than ciprofloxacin, amikacin and gentamicin. Different in vitro studies reveal that tigecycline and colistin are the only agents with consistent activity against MDR or panresistant MBL-producing isolates [27]. In the present study, all the MBL producing isolates of $E$. coli and $K$. pneumoniae were also sensitive to tigecycline and colistin by disc diffusion test and their MIC values were within the susceptible range. Among these isolates, the MIC values for colistin ranged from $0.125-0.5 \mu \mathrm{g} / \mathrm{mL}$ and for tigecycline ranged from $0.125-2.0 \mu \mathrm{g} / \mathrm{mL}$. However, the emergence of colistin and tigecycline resistance in Enterobacteriaceae is particularly menacing the future treatment options for bacterial infections $[28,29]$.

\section{Conclusions}

Our findings showed MBL production in a considerable number of $E$. coli and $K$. pneumoniae isolates with MDR and pandrug-resistant phenotypes. In the majority of hospitalized patients, MBL production in E. coli and K. pneumoniae is associated with increased mortality, morbidity and cost. Therefore, early detection of MBL producing $E$. coli and $K$. pneumoniae isolates has become indispensable for clinical microbiology laboratories. In absence of molecular detection techniques, the combined disc test provides a sensible choice for phenotypic detection of MBL production and can be implemented in clinical laboratory on a daily basis. In addition, routine surveillance of MBL producing bacteria is crucial for establishing appropriate empirical antimicrobial therapy and restraining their spread in hospital environment.

\begin{abstract}
Abbreviations
MBL: Metallo-beta-lactamase; E. coli: Escherichia coli; K. pneumoniae: Klebsiella pneumoniae; EDTA: Ethylenediaminetetraacetic acid; MHA: Mueller-Hinton agar; CLSI: Clinical Laboratory Standards Institute; MIC: Minimum inhibitory concentration; MHT: Modified Hodge test; ICU: Intensive care units; MDR: Multidrug-resistant.
\end{abstract}

\section{Competing interests}

The authors declare that they have no competing interests.

\section{Authors' contributions}

$A B, R S$ and $B K J$ conceived, participated in the design of the study, carried out the experimental work, evaluated the results and participated in drafting the manuscript. SNM \& KP collected the samples and characterized bacterial isolates. All authors have read and approved the submitted version of manuscript.

\section{Acknowledgements}

The authors would like to thank Prof. Giasuddin Ahmed (Department of Biotechnology, Gauhati University, India) for kindly providing the control strain E. coli strain NDM-1 EC27 and College of Medical Sciences, Bhartpur, Nepal for providing the necessary facilities to conduct this study.

Received: 12 April 2014 Accepted: 12 August 2014

Published: 21 August 2014

\section{References}

1. Rasheed JK, Kitchel B, Zhu W, Anderson KF, Clark NC, Ferraro MJ, Savard P, Humphries RM, Kallen AJ, Limbago BM: New Delhi metallo- $\beta$-lactamaseproducing Enterobacteriaceae, United States. Emerg Infect Dis 2013, 19:870-878.

2. Bebrone C: Metallo-beta-lactamases (classification, activity, genetic organization, structure, zinc coordination) and their superfamily. Biochem Pharmacol 2007, 74:1686-1701.

3. Ambler RP: The structure of beta-lactamases. Philos Trans R Soc Lond B Biol Sci 1980, 289:321-331.

4. Bush K, Jacoby GA: Updated functional classification of $\beta$-lactamases. Antimicrob Agents Chemother 2010, 54:969-976.

5. Patel G, Bonomo RA: "Stormy waters ahead": global emergence of carbapenemases. Front Microbiol 2013, 4:48.

6. Cornaglia G, Giamarellou H, Rossolini GM: Metallo- $\beta$-lactamases: a last frontier for $\beta$-lactams. Lancet Infect Dis 2011, 11:381-393.

7. Walsh TR: Emerging carbapenemases: a global perspective. Int I Antimicrob Agents 2010, 36(Suppl 3):S8-S14.

8. Collee JG, Miles RS, Watt B: Tests for Identification of Bacteria. In Mackie and McCartney Practical Medical Microbiology. 14th edition. Edited by Collee JG, Fraser AG, Marmion BP, Simmons A. New York: Churchill Livingstone; 1996:131-149.

9. Clinical Laboratory Standards Institute (CLSI): Performance Standards for Antimicrobial Susceptibility Testing; Twenty First Informational Supplement. Wayne PA: M 100-S21, CLSI; 2011.

10. Galani I, Kontopidou F, Souli M, Rekatsina PD, Koratzanis E, Deliolanis J, Giamarellou H: Colistin susceptibility testing by Etest and disc diffusion methods. Int J Antimicrob Agents 2008, 31:434-439.

11. Franklin C, Liolios L, Peleg AY: Phenotypic detection of carbapenem susceptible metallo-beta-lactamase-producing gram-negative bacilli in the clinical laboratory. J Clin Microbiol 2006, 44:3139-3144.

12. Mishra SK, Acharya J, Kattel HP, Koirala J, Rijal BP, Pokhrel BM: Metallobeta-lactamase producing gram-negative bacterial isolates. $J$ Nepal Health Res Counc 2012, 10:208-213.

13. Khanal S, Joshi DR, Bhatta DR, Devkota U, Pokhrel BM: $\beta$-lactamase-producing multidrug-resistant bacterial pathogens from tracheal aspirates of intensive care unit patients at national institute of neurological and allied sciences. Nepal ISRN Microbiol 2013, 2013:847569.

14. Yong D, Choi YS, Roh KH, Kim CK, Park YH, Yum JH, Lee K, Chong Y: Increasing prevalence and diversity of metallo-beta-lactamases in Pseudomonas spp., Acinetobacter spp., and Enterobacteriaceae from Korea. Antimicrob Agents Chemother 2006, 50:1884-1886.

15. Kumar S, Bandyopadhyay M, Mondal S, Pal N, Ghosh T, Bandyopadhyay M, Banerjee P: Tigecycline activity against metallo- $\beta$-lactamase-producing bacteria. Avicenna J Med 2013, 3:92-96.

16. Datta S, Wattal C, Goel N, Oberoi JK, Raveendran R, Prasad KJ: A ten year analysis of multi-drug resistant blood stream infections caused by Escherichia coli \& Klebsiella pneumoniae in a tertiary care hospital. Indian J Med Res 2012, 135:907-912.

17. Brusselaers $\mathrm{N}$, Vogelaers $\mathrm{D}$, Blot $\mathrm{S}$ : The rising problem of antimicrobial resistance in the intensive care unit. Ann Intensive Care 2011, 1:47.

18. Ramana KV, Rao R, Sharada CV, Kareem M, Reddy LR, Ratna Mani M: Modified Hodge test: a useful and the low-cost phenotypic method for detection of carbapenemase producers in Enterobacteriaceae members. J Nat Sci Biol Med 2013, 4:346-348.

19. Perry JD, Naqvi SH, Mirza IA, Alizai SA, Hussain A, Ghirardi S, Orenga S, Wilkinson K, Woodford N, Zhang J, Livermore DM, Abbasi SA, Raza MW: Prevalence of the faecal carriage of Enterobacteriaceae with NDM-1 carbapenemase at military hospitals in Pakistan, and the evaluation of two chromogenic media. J Antimicrob Chemother 2011, 66:2288-2294.

20. Bora A, Ahmed GU, Hazarika NK, Prasad KN, Shukla SK, Randhawa V, Sarma JB: Incidence of bla NDM-1 gene in Escherichia coli isolates at a tertiary care referral hospital in Northeast India. Indian J Med Microbio/ 2013, 31:250-256. 
21. Castanheira M, Deshpande LM, Mathai D, Bell JM, Jones RN, Mendes RE: Early dissemination of NDM-1-and OXA-181-producing Enterobacteriaceae in Indian hospitals: report from the SENTRY antimicrobial surveillance program, 2006-2007. Antimicrob Agents Chemother 2011, 55:1274-1278.

22. Deshmukh DG, Damle AS, Bajaj JK, Bhakre JB, Patwardhan NS: Metallo- $\beta$ lactamase-producing clinical isolates from patients of a tertiary care hospital. J Lab Physicians 2011, 3:93-97.

23. Seema K, Ranjan Sen M, Upadhyay S, Bhattacharjee A: Dissemination of the New Delhi metallo- $\beta$-lactamase-1 (NDM-1) among Enterobacteriaceae in a tertiary referral hospital in north India. J Antimicrob Chemother 2011, 66:1646-1647.

24. Stuart CJ, Leverstein-Van Hall MA, The Dutch Working Party on the detection of highly resistant microorganisms: Guidelines for the phenotypic screening and the confirmation of carbapenemases in Enterobacteriaceae. Int $J$ Antimicrob Agents 2010, 36:205-210.

25. Miriagou V, Cornaglia G, Edelstein M, Galani I, Giske CG, Gniadkowski M, Malamou-Lada E, Martinez-Martinez L, Navarro F, Nordmann P, Peixe L, Pournaras S, Rossolini GM, Tsakris A, Vatopoulos A, Canton R: Acquired carbapenemases in Gram-negative bacterial pathogens: detection and surveillance issues. Clin Microbiol Infect 2010, 16:112-122.

26. Nordmann P, Poirel L, Dortet L: Rapid detection of carbapenemaseproducing Enterobacteriaceae. Emerg Infect Dis 2012, 18:1503-1507.

27. Maltezou HC: Metallo-beta-lactamases in Gram-negative bacteria: introducing the era of pan-resistance. Int J Antimicrob Agents 2009, 33:405.e1-7.

28. Al-Qadheeb NS, Althawadi S, Alkhalaf A, Hosaini S, Alrajhi AA: Evolution of tigecycline resistance in Klebsiella pneumoniae in a single patient. Ann Saudi Med 2010, 30:404-407.

29. Kanj SS, Kanafani ZA: Current concepts in the antimicrobial therapy against resistant gram-negative organisms: extended-spectrum beta-lactamaseproducing Enterobacteriaceae, carbapenem resistant Enterobacteriaceae, and multidrug-resistant Pseudomonas aeruginosa. Mayo Clin Proc 2011, 86:250-259.

\section{doi:10.1186/1756-0500-7-557}

Cite this article as: Bora et al.: Incidence of metallo-beta-lactamase producing clinical isolates of Escherichia coli and Klebsiella pneumoniae in central Nepal. BMC Research Notes 2014 7:557.

\section{Submit your next manuscript to BioMed Central and take full advantage of:}

- Convenient online submission

- Thorough peer review

- No space constraints or color figure charges

- Immediate publication on acceptance

- Inclusion in PubMed, CAS, Scopus and Google Scholar

- Research which is freely available for redistribution 